\title{
Seroprevalence, clinical, and pathological characteristics of canine leishmaniasis in a central region of Colombia
}

\author{
Yenny Picón ${ }^{1,2}$, Geiner Almario ${ }^{1}$ \\ Victoria Rodríguez ${ }^{1}$, Noel Verjan Garcia ${ }^{1}$ \\ ${ }^{1}$ Immunobiology and Pathogenesis Research Group, Faculty of Veterinary Medicine, \\ University of Tolima, Altos de Santa Helena, Ibagué, Colombia \\ ${ }^{2}$ Master course in Public Health, \\ Autonomous University of Manizales, Manizales, Colombia \\ nverjang@ut.edu.co
}

Received: March 28, 2019 Accepted: February 3, 2020

\begin{abstract}
Introduction: Leishmaniasis is a zoonotic disease which is caused by protozoan parasites of the genus Leishmania. Canids are the most important reservoir of the parasites; however, limited data are available on the species of Leishmania prevalent in these animals and their impact on human health. The objective of this study was to estimate the seroprevalence of leishmaniasis in dogs from an inter-Andean region of Colombia during July 2016-July 2017, and to describe the clinical and histopathological features of the disease. Material and Methods: A total of 155 dogs were subjected to clinical examination and a serological test for detection of antibodies against Leishmania. Necropsy was carried out on positive animals and tissue samples were processed by routine histopathology. Results: Altogether 19 dogs were positive in the serological test, establishing a $12 \%$ seroprevalence of Leishmania. Clinical examination and necropsy revealed exfoliative and ulcerative dermatitis with haemorrhagic borders on the ears, head, nose, and legs. Histopathology revealed severe multifocal dermatitis with abundant Leishmania amastigotes within the cytoplasm of phagocytic cells, depletion of lymphocytes in lymphoid tissues, interstitial pneumonia, and interstitial nephritis. Tissue samples were positive for Leishmania by PCR. Conclusion: The macro- and microscopic changes correlated with the presence of Leishmania as established by serological test and PCR.
\end{abstract}

Keywords: Leishmania, antibodies, clinical examination, necropsy, histopathology.

\section{Introduction}

Leishmaniasis is a zoonotic disease endemic in tropical and subtropical areas of the world that include regions of Africa, India, Middle East, Southern Europe, and Central and South America (38). The disease is caused by trypanosomatid protozoans of the Leishmania genus, Leishmania and Viannia subgenera (23). The digenetic life cycle of the Leishmania parasite involves the transmission of flagellated promastigotes to mammal hosts during a sand-fly bite, where non-flagellated amastigotes develop within phagocytic mononuclear cells. These cells are taken up by the phlebotomine sand-fly vector during feeding and then develop into flagellated promastigotes, completing the life cycle $(17,22)$.
Leishmaniasis is manifested across a spectrum of clinical forms that encompasses subclinical (asymptomatic) infections, self-healing cutaneous lesions associated with sand-fly bites, and disseminated forms that include diffuse cutaneous leishmaniasis (CL), mucosal leishmaniasis (ML), and visceral leishmaniasis (VL) $(8,21)$. In America, VL is caused mainly by $L$. chagasi and L. amazonensis, whereas CL is caused by the L. mexicana species complex, which includes L. mexicana, L. amazonensis, and L. venezuelensis, and by the Viannia (V) subgenus (most notably L.V. braziliensis, L.V. panamensis, L.V. guyanensis, and L.V. peruviana). In addition, CL is also caused by $L$. major-like organisms and L. chagasi (23). In Europe, humans are considered the dominant reservoir for VL caused by $L$. donovani, and 
candidate vaccines have been developed with promising results $(6,9,18)$. However, none are effective at present, and the choice for therapy might be to target not only the host immune response but the parasite as well (38). In America, the domestic dog is known as the main animal reservoir host of L. infantum, whereas humans were described as incidental hosts (22); however, the question whether asymptomatic infected people can act as the reservoir host in urban areas in Brazil remains unresolved (16). Prevention of new infections in dogs can help to reduce or eventually stop the incidence of the disease in humans, and a number of candidate vaccines have been developed to prevent the disease in dogs (37). Currently, there are no approved commercial vaccines in Colombia to prevent leishmaniasis in dogs, and serologically positive animals are euthanised by the health authority.

Clinical cases of VL in humans have been estimated to be 0.2 to 0.4 million each year in the world, whereas CL cases reach 0.7 to 1.2 million cases, and despite underreporting, Colombia is among the ten countries with the highest estimated case counts together with Afghanistan, Algeria, Brazil, Iran, Syria, Ethiopia, North Sudan, Costa Rica, and Peru, which together represent $70 \%$ to $75 \%$ of global estimated CL incidence (5). In Panama, 3,000 new cases of tegumentary leishmaniasis occur per year, and 150 might progress to the mucocutaneous clinical form (30). In Colombia the frequency of the disease apparently reduced from 11,847 cases registered in 2016 to a total of 2,709 cases of all clinical forms of leishmaniasis during the first half-year of 2018, of which $98.56 \%(2,670 / 2,709)$ corresponded to CL, $1.21 \%(33 / 2,709)$ were ML, and $0.22 \%(6 / 2,709)$ were VL (3). In Colombia, CL in humans is associated most frequently with $L$. panamensis $(61.3 \%)$ and L. braziliensis $(27.1 \%)$, however, L. lainsoni $(0.6 \%)$, L. guyanensis $(0.9 \%)$, L. infantum chagasi (4\%), L. equatorensis $(0.6 \%), \quad$ L. mexicana $(2.1 \%)$, L. amazonensis $(2.8 \%)$, and L. colombiensis $(0.6 \%)$ were also found in lower proportions (36). There are no estimates of Leishmania cases in Colombian dogs, despite this animal constituting the main reservoir of Leishmania species in South America and canine infections often preceding the occurrence of human cases due to the close proximity of dogs to humans (18). A $17.2 \%$ prevalence of leishmaniasis in humans was reported in the inter-Andean region (the municipalities of Neiva, Algeciras, and Tello) of Huila, Colombia in 2002, and two years later 8,444 cases per 100,000 inhabitants $(8.4 \%)$ were reported in the Tolima inter-Andean region that includes the municipalities of Flandes and Chaparral. This suggests a migratory pattern of the Leishmania vector, the animal reservoirs or the affected human population from jungle areas such as Amazonas (an endemic area) to non-endemic Andean regions, thus increasing the peri-urban presence of the disease $(44,45)$.
In Europe, the seroprevalence of Leishmania in dogs varies significantly between countries; continentwide, an estimated 2.5 million $(16.7 \%)$ out of 15 million dogs were thought to be infected (33). Some infected dogs may eventually control the parasite and not develop the disease; however, others may develop progressive leishmaniasis that can be characterised by lymphadenopathy, found by Semião-Santos et al. in $89.3 \%(\mathrm{n}=28)$, skin lesions $(46 \%)$, onychogryphosis $(57 \%)$, somnolence $(25 \%)$, anorexia $(14.3 \%)$, and with less frequency cachexia, conjunctivitis, polyphagia, epistaxis, abnormal locomotion, weight loss, and vomiting (40). The mouse has been a useful animal model in the study of the pathogenesis of leishmaniasis (31); however, similarities in the clinical and pathological features between humans and dogs have allowed the use of dogs as an animal model in which to study the disease (1). Diagnosis of canine leishmaniasis is often conducted by serological tests that measure the presence of antibodies against particular Leishmania antigens; however, these tests may eventually fail to detect true positive samples or produce increased numbers of false-positive results due to cross-reactive antibodies, thus new technological advances have been proposed to overcome those limitations (34). PCR is becoming an important tool for the diagnosis of the disease in veterinary clinics (39) or monitoring the hair parasite load after medical treatment (13), and distinct non-invasively taken tissue samples such as conjunctival swabs are being evaluated for diagnosis of canine leishmaniasis (24). As a preliminary attempt to understand the epidemiology of canine leishmaniasis in a central region of Colombia, this study was designed to estimate the seroprevalence of antibodies to its antigens in dogs located in close proximity to people that had been diagnosed with the disease. Histopathological detection and molecular detection of the parasite DNA by PCR were also conducted.

\section{Material and Methods}

Location. This study was carried out in the municipalities of Guamo, Ortega, Flandes, Coyaima, and Melgar (the south-western part of Tolima Department) and the municipality of Neiva (Huila Department), located in proximity to the Magdalena valley in the middle western part of Colombia between the Andean mountain subranges (Cordillera Central and Cordillera Oriental). These regions are delimited by coordinates $4^{\circ} 55^{\prime}$ north latitude and $75^{\circ} 07^{\prime}$ west longitude and $2^{\circ} 59^{\prime} 55^{\prime \prime}$ north latitude and $75^{\circ} 18^{\prime} 16^{\prime \prime}$ east longitude. The altitude varies from $442 \mathrm{~m}$ above sea level in Neiva to $320 \mathrm{~m}$ in Guamo, Tolima. The average temperature varies from $27-29^{\circ} \mathrm{C}$. A number of small affluent rivers such the Las Ceibas River and Gold River among others cross those municipalities and flow into the main stream of the Magdalena River. In this region a number of cases of human 
leishmaniasis have been reported by the health authority.

Study design and epidemiological variables. A descriptive cross-sectional study was designed to estimate the preliminary seroprevalence of leishmaniasis in canines from some municipalities of Tolima and Huila, Colombia, where active surveillance of human leishmaniasis is conducted by the health authority due to the increasing number of clinical cases of the disease. With this purpose, an instrument (a survey) was conceived and executed to obtain epidemiological variables from the environment of pet dogs and the living conditions of their owners. These owners had previously been informed of and signed consent to be included in the study and the data retained from it. The epidemiologic variables included: a) characteristics of the housing: department and home municipality, material from which the home is constructed, method of disposal of excreta and refuse, presence of sick people, presence of house plants, proximity to streams or pipes, presence of other domestic animals that can be reservoirs, presence of pests (insects and rodents) in the extra- and peridomicile; and b) characteristics of the canines: sex, age by dental chronometry, length of stay in the home, clinical signs if any, anamnesis, displacements (trips) of the animal in the last six months or within a year, and its physical condition. The data were collected during two visits to the pets' owners, input to Excel (Microsoft Corporation, Redmond, WA, USA), and processed by descriptive analysis.

Sampling and sample size. Sampling in the municipalities of the Tolima Department was performed in peri-urban areas where the surveillance system indicated the presence of human leishmaniasis and potentially infected dogs, whereas sampling in the municipality of Neiva was conducted in communes 8 , 9 , and 10, which corresponded to locations established by the health authority as peri-focal areas of human leishmaniasis. The population constituted a total of 155 dogs, and $95.48 \%$ (148) of the sampled animals were symptomatic. Blood samples $(5 \mathrm{~mL})$ were taken by puncture of the cephalic vein, collected in sterile redcap tubes with BD Vacutainer equipment (Beckton Dickinson, Franklin Lakes, NJ, USA), and kept in the tubes at $4^{\circ} \mathrm{C}$ for $5 \mathrm{~h}$ to allow complete coagulation. Blood serum was obtained after centrifugation at $3,000 \mathrm{rpm}$ for $10 \mathrm{~min}$. Haemograms were also conducted on the same day and total blood protein levels established. All dogs with serological reactivity to leishmaniasis were included, and those reactive canines that could not be identified at the time of the second visit due to death or loss of housing were excluded. Serologically positive dogs were subjected to euthanasia according to Colombian law (Law 576 of 2000 , article 19, paragraph 2), followed by a complete necropsy protocol, where tissue samples from all organs were collected and fixed in $10 \%$ buffered formalin.
Clinical examination. All dogs were given a thorough clinical examination performed by a veterinarian clinician adjunct to the municipal health authority or by the veterinarian researcher in charge of the study. Clinical signs compatible with leishmaniasis were recorded. They included dermatological lesions, eye changes, weight loss, apathy, nervous signs, and increased size of lymph nodes or spleen at palpation. Data were recorded during the survey and refined during a second visit. Blood samples were taken during the clinical examination.

Serological test for leishmaniasis. Blood serum collected from asymptomatic and symptomatic dogs was used for the detection of specific anti-Leishmania antibodies by using the Kalazar Detect kit (InBios, Seattle, WA, USA). This technique was carried out at the Public Health Laboratory of Huila and Tolima. The K39 test strip was used, which exploits a purified recombinant antigen from Leishmania and detects antiLeishmania $\operatorname{IgG}$ antibodies by immunochromatography after $5 \mathrm{~min}$ of exposure to serum. Positive samples typically show 2 immunoreactive bands, whereas negative samples show a single band.

Necropsy and histopathology. Canines that were positive in the serological test for leishmaniasis were euthanised by administering sodium pentobarbital (Euthanex, INVET, Bogota, Colombia) intravenously at doses of $1 \mathrm{~mL}$ per $5 \mathrm{~kg}$ of b.w. A complete necropsy was conducted, and $1-3 \mathrm{~cm}^{3}$ tissue samples were collected and fixed in $10 \%$ buffered formalin. Fixed tissues were embedded in paraffin, and $5 \mu \mathrm{m}$ thick tissue sections were cut and processed by routine haematoxylin-eosin (H\&E) staining. Slides were analysed under a light microscope, and about 100 fields were observed per slide. Amastigote forms of Leishmania were visualised by using a CX43 light microscope (Olympus Life Science Solutions, Tokyo, Japan) with a $100 \times$ objective lens with immersion oil.

Polymerase chain reaction. Total genomic DNA was extracted from freshly collected tissues (skin, lymph nodes, and spleen) during necropsy using the Easy-DNA extraction kit (Invitrogen, Waltham, MA, USA). The DNA was used as a template in a conventional PCR to amplify a 314 bp DNA fragment of the internal transcribed region (ITS1) of the small subunit ribosomal DNA gene of Leishmania spp. using the primers L5.8S 5'-TGATACCACTTATCGCACTT-3' and LITSR 5'-CTGGATCATTTTCCGATG-3' described by Al-Nahhas and Kaldas in 2013 (4). The PCR reaction was performed in $25 \mu \mathrm{L}$ of total volume using the AccuPrime ${ }^{\mathrm{TM}}$ Taq DNA polymerase System (Thermo Fisher Scientific, Waltham, MA, USA), containing $2.5 \mu \mathrm{L}$ of $\mathrm{MgCl}_{2}, 2.5 \mu \mathrm{L}$ of $10 \times$ buffer, $1 \mu \mathrm{L}$ of each primer $(10 \mu \mathrm{M}), 8 \mathrm{mM}$ of deoxyribonucleotide triphosphates (dNTPs), $0.5 \mu \mathrm{L}$ of Taq DNA polymerase, and $2 \mu \mathrm{L}$ of DNA template. Amplification was carried out in a T-100 thermal cycler (Bio-Rad, Hercules, CA, USA) with the following PCR conditions: an initial denaturation step at $95^{\circ} \mathrm{C}$ for 
$3 \mathrm{~min}$, followed by 37 denaturation cycles at $94^{\circ} \mathrm{C}$ for $30 \mathrm{~s}$, annealing at $55^{\circ} \mathrm{C}$ for $30 \mathrm{~s}$, extension at $72^{\circ} \mathrm{C}$ for $1 \mathrm{~min}$, and a final extension step at $72^{\circ} \mathrm{C}$ for $5 \mathrm{~min}$. The amplification products were separated by $1.5 \%$ agarose gel electrophoresis at 100 volts for $30 \mathrm{~min}$ and stained with ethidium bromide. As controls, the DNA from L. braziliensis, L. amazonensis, and L. infantum fresh culture were used according to the recommendations of the National Institute of Health of Colombia (36).

Statistical analysis. Most of the epidemiologic variables were processed by descriptive statistics. Associations between the epidemiological variables and positivity to Leishmania spp. were evaluated by a Chi-square test $\left(\chi^{2}\right)$, whereas the strength of association was calculated by the odds ratio (OR) with a level of significance of $p<0.05$. The SPSS Statistics 2.0 (IBM, Armonk, NY, USA) software was used.

Ethical considerations. The study complied with the Colombian regulations set forth in Law 84 of 1989 , article 17, section $f$, which mandates the immediate slaughter of animals that represent a threat to public health. It also complied with Resolution 8430 of 1999 (title $\mathrm{V}$, article 87), wherein it is mandated that the euthanasia of the animals will be carried out with appropriate anaesthetics approved by the veterinary association. Pet owners were requested to sign an informed consent when animals were found positive for the presence of anti-Leishmania antibodies by the serological test. The minimum pain and the highest conditions of animal welfare, analgesia, and respect were provided as the dogs were euthanised. The study was approved by the Bioethics Committee of the
Autonomous University of Manizales, in compliance with Colombian law.

\section{Results}

A total of 155 dogs' samples were analysed by the Kalazar serological test to detect antibodies against Leishmania parasites. By geographical origin, 95.5\% $(\mathrm{n}=148)$ were from Huila Department and $4.5 \%(n=7)$ from Tolima Department. The Kalazar serological test detected 19 symptomatic dogs out of 155 positive for anti-Leishmania antibodies, establishing a seroprevalence of $12 \%$ leishmaniasis in dogs for these regions during the period 2016-2017. Fourteen positive dogs (74\%) were from the municipality of Neiva, Huila, and five (26\%) were from the municipalities of Guamo (1), Coyaima (1), Ortega (1), Melgar (1), and Flandes (1), Tolima. Complete sampling and tissue processing was possible only in 11 serologically reactive dogs. The remaining eight dogs (five from Huila and three from Tolima) were not available at the time of the second visit to the owner because they were apparently lost. The epidemiological variables of sex $(7.385, p=0.007)$, presence of pests $(8.25, p=0.004)$, presence of dogs per house (8.93; $\mathrm{p}=0.003)$, and recent trips $(5.123, \mathrm{p}=0.024)$ were significantly associated $(\mathrm{p}<0.05)$ with positivity for leishmaniasis. The other epidemiological variables of construction material of the housing, presence of house plants, or proximity to streams or pipes did not associate with positivity for leishmaniasis (Table 1).

Table 1. Epidemiological variables associated with leishmaniasis in dogs from a central region of Colombia (July 2016-July 2017)

\begin{tabular}{|c|c|c|c|c|c|c|c|c|}
\hline Variables & Tolima & $\%$ & Huila & $\%$ & Total & $\%$ & OR & $\mathrm{P}<0.05$ \\
\hline \multicolumn{9}{|c|}{ Characteristics of housing } \\
\hline Bamboo & 1 & 33.3 & 0 & 0.0 & 1 & 9.1 & & \\
\hline Brick & 1 & 33.3 & 4 & 50.0 & 5 & 45.5 & & \\
\hline Wood & 0 & 0.0 & 3 & 37.5 & 3 & 27.3 & & \\
\hline Gaudua & 1 & 33.3 & 1 & 12.5 & 2 & 18.2 & 10.5 & 0.398 \\
\hline Sick people & 0 & 0.0 & 2 & 25.0 & 2 & 18.2 & 7 & 0.221 \\
\hline Garbage & 1 & 33.3 & 4 & 50.0 & 5 & 45.5 & 7 & 0.221 \\
\hline Reservoirs & 3 & 100.0 & 8 & 100.0 & 11 & 100.0 & 8.93 & 0.003 \\
\hline House plants & 3 & 100.0 & 7 & 87.5 & 10 & 90.9 & 7 & 0.221 \\
\hline Stream or pipes & 2 & 66.7 & 7 & 87.5 & 9 & 81.8 & 2.91 & 0.713 \\
\hline Pests & 2 & 66.7 & 7 & 87.5 & 9 & 81.8 & 8.25 & 0.004 \\
\hline \multicolumn{9}{|c|}{ Characteristics of canine } \\
\hline Male & 3 & 100.0 & 7 & 87.5 & 10 & 90.9 & 7.35 & 0.007 \\
\hline Female & 0 & 0.0 & 1 & 12.5 & 1 & 9.1 & & \\
\hline Age & 6.3 & $3-11$ & 3.6 & $2-5$ & 4.4 & $2-11$ & 14 & 0.173 \\
\hline Cachexia & & & & & & & 2.917 & 0.713 \\
\hline \multicolumn{9}{|l|}{ Normal } \\
\hline Trips & 1 & 33.3 & 4 & 50.0 & 5 & 45.5 & 5.125 & 0.024 \\
\hline \multicolumn{9}{|l|}{ Symptomatology } \\
\hline Anamnesis & 3 & 100 & 7 & 87.5 & 10 & 90.9 & 4.95 & 0.421 \\
\hline
\end{tabular}


The haemogram showed an increased value of eosinophils characteristic of a parasitic disease in some animals, but it was not the rule. The necropsy revealed multiple alopecic areas of the skin, focal exfoliative dermatitis, and skin ulcers, some with haemorrhagic borders, located on the ears, nose, head, neck, and shoulders (Figs 1 A, B). The examination also found moderate levels of onychogryphosis, splenomegaly with an irregular surface, and numerous pale nodules that had ulcerated in some cases (Figs $1 \mathrm{C}, \mathrm{D}$ ), and ulcerative nodules were also present on the lung surface (Figs 1 E, F). Histopathological analyses revealed the exfoliative dermatitis to be characterised by a mononuclear cell infiltrate with abundant Leishmania amastigotes within the cytoplasm of leukocytes including histiocytes and macrophages, and they also found depletion of lymphocytes in lymph nodes with abundant parasites in the cytoplasm of macrophages. In some cases interstitial pneumonia, interstitial nephritis, and necrosis were also present (Fig. 2). A lymphoplasmacytic infiltrate was predominantly seen in the intestinal lesions of some dogs. PCR analysis detected a 314 bp fragment of a Leishmania spp. RNA gene in skin, lymph nodes, and spleen samples (Fig. 3) from most dogs.
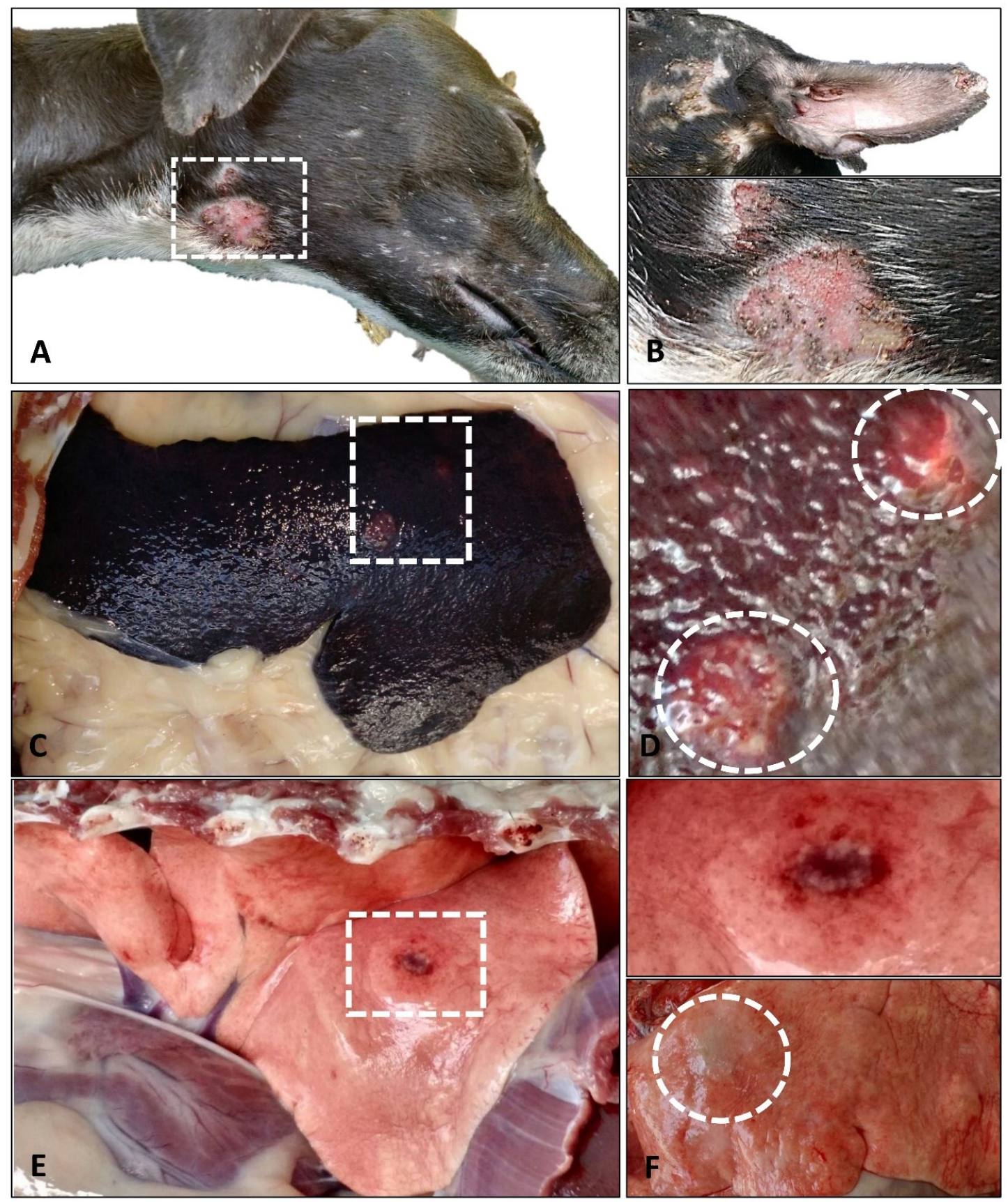

Fig. 1. Macroscopic lesions in dogs with leishmaniasis in a central region of Colombia. A, B - skin lesions (exfoliative and ulcerative dermatitis) on the head, neck, and ears (Fig. B lower panel is a magnification of the delineated square in A). C, $\mathrm{D}$ - splenomegaly with nodules protruding on the irregular surface of the organ (Fig. D is a magnification of the delineated square in fig. C). E, F upper panel - ulcerated nodule in the lung (Fig. F upper panel is a magnification of the delineated square in Fig. E). F lower panel - a non-ulcerated nodule protruding from the surface of the lung of a different dog 


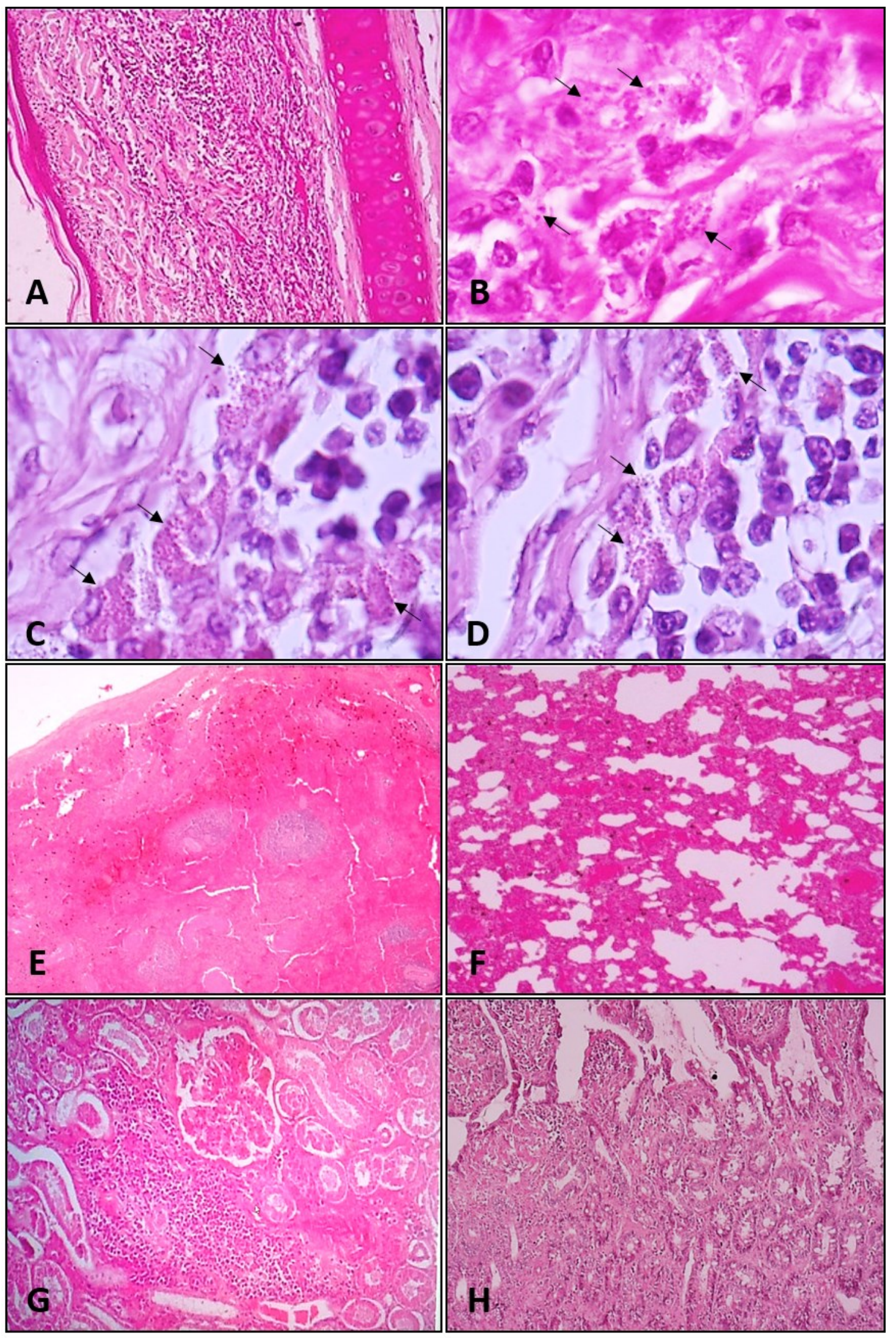

Fig. 2. Microscopic lesions in dogs with leishmaniasis in a central region of Colombia. A, B - auricular dermatitis associated with intracellular amastigotes present within macrophages (arrows). C, D - lymphadenitis showing abundant promastigotes parasites (arrows) within phagocytic mononuclear cells. E - spleen with severe depletion of lymphocytes (white pulp) and apparent hyperplasia of red pulp. F - lung with moderate to severe interstitial pneumonia. $\mathrm{G}$ - kidney showing interstitial nephritis with tubular degeneration. $\mathrm{H}$ - small intestine showing lymphoplasmacytic infiltrate 


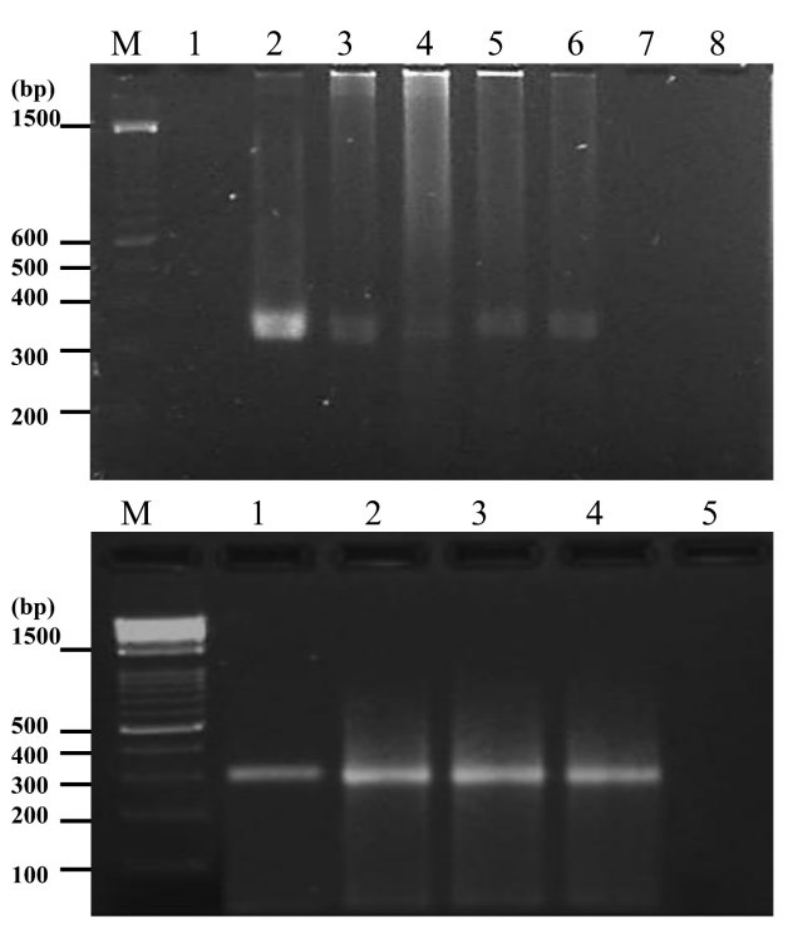

Fig. 3. $P C R$ Amplification of a 314 bp DNA fragment of a Leishmania RNA gene. Upper panel - M:100 bp ladder. Lane 1 negative control (without template DNA). Lane 2 - positive control of $L$. amazonensis. Lane 3 - spleen. Lane 4 - skin. Lanes 5, 6 lymph nodes. Lane 7 - positive control of $L$. infantum. Lane $8-$ positive control of $L$. braziliensis. Lower panel - M: 100 bp ladder. Lane 1 - positive control of $L$. amazonensis. Lanes 2-5 - spleen samples from three serological positive dogs

\section{Discussion}

This study is the first description of the clinical and pathological findings in pet dogs with leishmaniasis from a central region of Colombia, where a seroprevalence of $12 \%$ was estimated for the period of July 2016-July 2017. This was lower than the 22\% prevalence reported by the regional health authority of Huila for the period 2008-2014 (45), suggesting an apparent reduction of seropositive animals or otherwise a reduction in the number of notifiable clinical cases. Supporting the second assumption, a number of pet owners did not cooperate with the euthanasia of their seropositive dogs, which were not available on the second visit. A $12 \%$ seroprevalence of leishmaniasis in dogs in this region of Colombia is lower than the seroprevalence $(22 \%)$ of VL in dogs from Brazil, where a higher number of animals were evaluated $(n=5,822)$ and where the re-emergence of the disease appears to be a consequence of the discontinuation of control programmes (27). The prevalence reported in this study was higher than the seroprevalence $(6.31 \%)$ of canine leishmaniasis reported in Portugal in 2009, where a total of 3,974 dogs were analysed (14). In that country, the seroprevalence of the disease appeared to have increased over time since $3.9 \%(141 / 3,614)$ was registered in 1995 (40). Similarly, the seroprevalence of canine leishmaniasis caused by L. infantum ranged from $4.9 \%$ to $12.6 \%$ in dogs from a public kennel in Bologna, Italy (10), whereas a $16 \%$ VL incidence rate was reported in dogs from Iran, where infection was significantly more prevalent in older dogs $(16-31 \%$ in dogs more than 3 years old) than younger ones $(10 \%-$ $12 \%$ in dogs up to 2 years old), in stray dogs than owned dogs ( $10 \%$ vs. $6 \%$, respectively), and in rural dogs than urban ones ( $36 \%$ vs. 19\%, respectively) (41). The prevalence of canine leishmaniasis varies considerably between different regions and depending on the serological test used in the diagnosis; for example in Algeria, the seroprevalence of leishmaniasis was $11.7 \%$ in stray dogs, $9.7 \%$ in national guard dogs, and $5.9 \%$ in farm dogs using the immunofluorescence antibody test (IFAT) (2). Since only sporadic case reports of leishmaniasis in dogs from Colombia were found in the published literature, this study constitutes preliminary base line data for the seroprevalence of Leishmania in owned dogs in a central region of Colombia.

Leishmaniasis is endemic in South America, and epidemiological data are available from some of its countries: for example in Brazil, canine VL is caused mainly by $L$. infantum, which was detected in blood $(28.05 \%)$, lymph nodes $(57.32 \%)$, and bone marrow $(69.51 \%)$ samples from stray dogs in a VL-endemic area (12). L. infantum (L. chagasi) is also responsible for leishmaniasis in domestic cats in Brazil (32). In addition, two genotypes of $L$. infantum (LiAl and LiA2) identified by sequencing the cytochrome B (cytb) gene of Leishmania were associated with canine leishmaniasis in Argentina (11). In this study, L. amazonensis DNA was detected by PCR from various organs of infected dogs, whereas the samples were apparently negative for $L$. braziliensis and L. infantum. Although the impact of any particular Leishmania parasite circulating in dogs from those regions of Colombia is currently unknown, L. amazonensis and L. panamensis were previously reported in humans and dogs from the inter-Andean valley of this country (36). In contrast, a previous epidemic outbreak of leishmaniasis in 72 guard dogs from the Colombian military personnel that accompanied soldiers on a mission to destroy illicit crops in the jungle was caused by L. braziliensis and L. panamensis (44). In addition, L. amazonensis is known to be responsible for at least $8 \%$ of tegumentary cases of human leishmaniasis in northern and northeastern Brazil (43). In another region of Brazil (Vila Operaria Buerarema Bahia), 50.3\% (147/292) seropositivity in dogs was detected by immunofluorescence assay, and only 3.66\% (10/273) were positive for L. braziliensis by PCR, whereas all samples were negative for L. infantum chagasi (28). Therefore, the results of this research strengthen the need to conduct robust epidemiological studies on the species and distribution of Leishmania causing canine leishmaniasis and its direct association with cases of the disease in humans. However, since culling of 
infected dogs is considered unethical and found to be an inefficacious strategy to control or prevent the disease in humans (35), governmental authorities should implement more precise diagnostic methods for early detection of infected dogs, and commission clinical evaluation of available preventive vaccines or the development of strain-specific vaccines in Colombia.

The majority of serologically positive dogs in this study manifested skin lesions (alopecia, exfoliative and ulcerative dermatitis) and onychogryphosis associated with leishmaniasis, but no clear lymphadenopathy was evident despite the abundant parasitic burden in these organs (Fig. 2). Some dogs presented a distended abdomen and emaciation that correlated with splenomegaly. These findings are consistent with reported clinical signs in canine leishmaniasis caused by $L$. infantum in Argentina, where infected canids most frequently showed skin abnormalities $(87 \%)$ that included alopecia $(54.2 \%)$ or secondary skin lesions (47.9\%), lymphadenopathy (89.6\%), and emaciation (75\%) (11). In addition, in a study from 28 seropositive dogs in Portugal, the most prevalent lesions were lymphadenopathy $(89.3 \%, \mathrm{n}=25)$, onychogryphosis $(57.1 \%, \mathrm{n}=16)$, and skin lesions $(46.4 \%, \mathrm{n}=13)(40)$. Spontaneous cases of canine leishmaniasis $(n=22)$ in Greece were predominantly characterised by exfoliative dermatitis (90.9\%), ulcerations (63.6\%), onychogryphosis $(54.5 \%)$, sterile pustular dermatitis (13.6\%), and paronychia (13.6\%) (25).

The predominant skin (skin ulcers and dermatitis) and lymphoid tissue (lymphocyte depletion) lesions in dogs observed in this study were characterised by the presence of abundant Leishmania spp. amastigotes in the cytoplasm of phagocytic cells (Fig. 2), whereas the parasite was scarce in other inflamed organs such as the spleen, lungs, kidneys, and intestines despite the evident inflammatory reaction characterised by mononuclear cells (lymphoplasmacytic infiltrate including lymphocytes, macrophages, and plasma cells). Skin lesions were predominant on the head, neck, and ears, where dissemination of the parasite seems to be favoured by self-mutilation. These results are in agreement with previous reports of leishmaniasis in dogs from Spain (19) and Brazil, where skin and lymph node lesions were predominant and Leishmania amastigotes were observed in $19 \%$ of infected dogs, amastigote frequency increasing to $36 \%$ in dogs with Leishmania and Ehrlichia coinfection (7). The authors also reported structural disorganisation of white pulp in the spleen and both white pulp hypoplasia and hyperplasia in some dogs. In contrast, in this study only lymphoid tissue depletion (hypoplasia) was evident (Fig. 2). Lymphadenopathy is a hallmark of canine leishmaniasis and is characterised by an increased number and size of lymphoid follicles and marked hypertrophy and hyperplasia of medullary macrophages. However, differences in the involvement of different peripheral lymph nodes exist, such as in cases finding cervical and popliteal lymph nodes more severely affected than axillary ones in dogs with visceral leishmaniasis in Brazil (29).

Less frequently, interstitial pneumonia, interstitial nephritis (Fig. 2), glomerulonephritis, and tubular degeneration were present in some dogs of this study. These changes in the kidneys have been more accurately described in dogs with VL in Brazil, where glomerulonephritis was found in 100\% (55/55 dogs), interstitial alteration in $96.3 \%$, and tubular changes in $78.18 \%$ (15), and chronic interstitial pneumonia was also described in canine leishmaniasis in Brazil $(20,42)$.

In conclusion, this study for the first time established a preliminary seroprevalence of $12 \%$ for leishmaniasis in dogs from various municipalities of Tolima and Huila, Colombia. The disease was characterised by prominent skin and visceral lesions, and histological analysis revealed dermatitis and lymphadenopathy associated with abundant amastigote parasites within phagocytic cells and less frequent interstitial pneumonia and interstitial nephritis. Finally, the disease was associated with the presence of Leishmania spp. DNA by conventional PCR. More robust epidemiological studies are needed to understand the involvement of other Leishmania species in dogs and humans and to enable the development of species-specific strategies of prevention.

Conflict of Interests Statement: The authors declare that there is no conflict of interests regarding the publication of this article.

Animal Rights Statement: The experiments on animals were conducted in accordance with the local Ethical Committee laws and regulations as regards care and use of experimental animals.

Financial Disclosure Statement: This research was funded by grants from the Central Research Office of the University of Tolima awarded to Noel Verjan (Project No. 450114).

\section{References}

1. Abbehusen M.M.C., Almeida V.A., Solcà M.S., Pereira L.S., Costa D.J., Gil-Santana L., Bozza P.T., Fraga D.B.M., Veras P.S.T., dos Santos W.L.C., Andrade B.B., Brodskyn C.I.: Clinical and immunopathological findings during long term follow-up in Leishmania infantum experimentally infected dogs. Sci Rep 2017, 7, 15914, doi: 10.1038/s41598-017-15651-8.

2. Adel A., Saegerman C., Speybroeck N., Praet N., Victor B., De Deken R., Soukehal A., Berkvens D.: Canine leishmaniasis in Algeria: true prevalence and diagnostic test characteristics in groups of dogs of different functional type. Vet Parasitol 2010, 172, 204-213, doi: 10.1016/j.vetpar.2010.05.009.

3. Agudelo Chivata N.J.: Informe de evento Leishmaniasis, Colombia, Semestre I de 2018. (Leishmaniasis report for Colombia for Jan-Jun 2018 - in Spanish) Instituto Nacional de Salud, Ministerio de Salud, Colombia, 2018. pp. 1-22. 
4. Al-Nahhas S.A., Kaldas R.M.: Characterization of Leishmania species isolated from cutaneous human samples from central region of Syria by RFLP analysis. ISRN parasitol 2013, 308726, doi:10.5402/2013/308726.

5. Alvar J., Vélez I.D., Bern C., Herrero M., Desjeux P., Cano J., Jannin J., Den Boer M., The WHO Leishmaniasis control team: Leishmaniasis Worldwide and Global Estimates of Its Incidence. PLoS ONE 2012, 7, 1-12, e35671, doi: 10.1371/journal.pone.0035671.

6. Anand S., Madhubala R.: Genetically engineered ascorbic aciddeficient live mutants of Leishmania donovani induce long lasting protective immunity against visceral leishmaniasis. Sci Rep 2015, 5, 10706, doi: 10.1038/srep10706.

7. Andrade G.B., Gomes Barreto W.T., dos Santos L.L., Ribeiro L.R.R., Macedo G.C. de, Sousa K.C.M. de, André M.R., Machado Z.R., Herrera H.M.: Pathology of dogs in Campo Grande, MS, Brazil naturally co-infected with Leishmania infantum and Ehrlichia canis. Braz J Vet Parasitol 2014, 23, 509-515.

8. Aronson N., Herwaldt B.L., Libman M., Pearson R., LopezVelez R., Weina P., Carvalho E.M., Ephros M., Jeronimo S., Magill A.: Diagnosis and treatment of leishmaniasis: Clinical Practice Guidelines by the Infectious Diseases Society of America (IDSA) and the American Society of Tropical Medicine and Hygiene (ASTMH). CID 2016, 63, e202-e264.

9. Avishek K., Kaushal H., Gannavaram S., Dey R., Selvapandiyan A., Ramesh V., Negi N.S., Dubey U.S., Nakhasi H.L., Salotra P.: Gene deleted live attenuated Leishmania vaccine candidates against visceral leishmaniasis elicit pro-inflammatory cytokines response in human PBMCs. Sci Rep 2016, 6, 33059, doi: 10.1038/srep33059.

10. Baldelli R., Piva S., Salvatore D., Parigi M., Melloni O., Tamba M., Bellini R., Poglayen G.: Canine leishmaniasis surveillance in a northern Italy kennel. Vet Parasitol 2011, 179, 5761, doi: 10.1016/j.vetpar.2011.01.052.

11. Barroso P.A., Nevot M.C., Hoyos C.L., Locatelli F.M., Lauthier J.J., Ruybal P., Cardozo R.M., Russo P.D., Vassiliades C.N., Mora M.C., Estevez J.O., Hashiguchi Y., Korenaga M., Basombrio M.A., Marco J.D.: Genetic and clinical characterization of canine leishmaniasis caused by Leishmania (Leishmania) infantum in northeastern Argentina. Acta Trop 2015, 150, 218-223, doi: 10.1016/j.actatropica.2015.08.007.

12. Coiro C.J., Coelho L.G.G., da Silva R.C., Langoni H.: Molecular characterization of Leishmania spp. isolated from Brazilian stray dogs from an endemic area for canine visceral leishmaniasis. Vet Parasitol Regional Studies and Reports 2017, 7, 9-13, doi: 10.1016/j.vprsr.2016.11.005.

13. Corpas-López V.G., Merino-Espinosa C., Acedo-Sánchez V., Díaz-Sáez F., Morillas-Márquez J., Martın S.: Hair parasite load as a new biomarker for monitoring treatment response in canine leishmaniasis. Vet Parasitol 2016, 223, 20-25, doi:10.1016/ j.vetpar.2016.04.001

14. Cortes S., Vaz Y., Neves R., Maia C., Cardoso L., Campino L.: Risk factors for canine leishmaniasis in an endemic Mediterranean region. Vet Parasitol 2012, 189, 189-196, doi: 10.1016/j.vetpar.2012.04.028

15. Costa F.A.L., Goto H., Saldanha L.C.B., Silva S.M.M.S., Sinhorini I.L., Silva T.C., Guerra J.L.: Histopathologic patterns of nephropathy in naturally acquired canine visceral leishmaniasis. Vet Pathol 2003, 40, 677-684.

16. Dantas-Torres F., Brandão-Filho P.S.: Visceral leishmaniasis in Brazil: revisiting paradigms of epidemiology and control. Rev Inst Med trop S Paulo 2006, 48, 151-156

17. De Luca P.M., Mayrink W., Alves C.R., Coutinho S.G., Oliveira M.P., Bertho A.L., Toledo V.P., Costa C.A., Genaro O., Mendonca S.C.F.: Evaluation of the stability and immunogenicity of autoclaved and nonautoclaved preparations of a vaccine against American tegumentary leishmaniasis. Vaccine 1999, 17, 1179-1185.

18. Duthie M.S., Pereira L., Favila M., Hofmeyer K.A., Reed S.J., Metangmo S., Townsend S., Laurance J.D., Picone A., Misquith A.,
Hossain F., Ghosh P., Khan M.A.A., Guderian J., Bailor H.R., Liang H., Vergara J., Oliveira F., Howard R.F., Kamhawi S., Mondal D., Coler R.N., Valenzuela J.G., Reed S.G.: A defined subunit vaccine that protects against vector-borne visceral leishmaniasis. Vaccines 2017, 2, 23, doi: 10.1038/s41541-0170025-5.

19. Ferrer L., Rabanal R., Fondevila D., Ramos J.A., Domingo M.: Skin lesions in canine leishmaniasis. J small Anim Pract 1988, 29, 381-388.

20. Gonçalves R., Tafuri W., Melo M.N., Raso P., Tafuri W.L.: Chronic interstitial pneumonitis in dogs naturally infected with Leishmania (Leishmania) chagasi: a histopathological and morphometric study. Rev Inst Med trop S Paulo 2003, 45, 153-158.

21. González de la Fuente S., Peiró Pastor R., Rastrojo A., Moreno J., Carrasco Ramiro F., Requena J.M., Aguado B.: Resequencing of the Leishmania infantum (strain JPCM5) genome and de novo assembly into 36 contigs. Sci Rep Archiving 2017, 7, 1-10, doi: 10.1038/s41598-017-18374-y.

22. Handman E.: Leishmaniasis: current status of vaccine development. Clin Microbiol Rev 2001, 14, 229-243, doi: 10.1128/CMR.14.2.229-243.2001.

23. Herwaldt B.L.: Leishmaniasis. Lancet 1999, 354, 1191-1199.

24. Karakus M., Toz S., Ertabaklar H., Pas S., Atasoy A., Arserim S.K., Olgen M.K., Alkan M.Z., Durrant C., Ozbel Y.: Evaluation of conjunctival swab sampling in the diagnosis of canine leishmaniasis: a two-year follow-up study in Cukurova Plain, Turkey. Vet Parasitol 2015, 214, 295-302, doi: 10.1016/j.vetpar.2015.09.009

25. Koutinas A., Scott A.W., Kantos V., Lekkas S.: Skin lesions in canine leishmaniasis (Kala-Azar): A clinical and histopathological study on 22 spontaneous cases in Greece. Vet Dermatol 199, 3, 121-130, doi:10.1111/j.13653164.1992.tb00158.x

26. Lainson R., Shaw J.J.: Epidemiology and ecology of leishmaniasis in Latin-America. Nature 1978, 273, 595-600.

27. Leal G.G.de A., Carneiro M., Pinheiro A. da C., Marques L.A., Ker H.G., Reis A.B., Coura-Vital W.: Risk profile for Leishmania infection in dogs coming from an area of visceral leishmaniasis reemergence. Prev Vet Med 2018, 150, 1-7.

28. Leça Júnior N.F., Guedes P.E.B., Santana L.N., Almeida V. dos A., Carvalho F.S., Albuquerque G.R., Wenceslau A.A., Munhoz A.D., Silva F.L.: Epidemiology of canine leishmaniasis in southern Bahia, Brazil. Acta Trop 2015, 148, 115-119, doi: 10.1016/j.actatropica.2015.04.008.

29. Lima W.G., Michalick M.S.M., Melo M.N., de Tafuri W.L., Tafuri W.L.: Canine visceral leishmaniasis: a histopathological study of lymph nodes. Acta Tropica 2004, 92, 43-53, doi: 10.1016/j.actatropica.2004.04.007.

30. Llanes A., Restrepo C.M., Del Vecchio G., Anguizola F.A., Lleonart R.: The genome of Leishmania panamensis: insights into genomics of the L. (Viannia) subgenus. Sci Rep 2015, 5, 8550, doi: $10.1038 /$ srep08550.

31. Melo Guilherme D., Goyard S., Fiette L., Boissonnas A., Combadiere C., Machado G.F., Minoprio P., Lang T.: Unveiling cerebral leishmaniasis: parasites and brain inflammation in Leishmania donovani infected mice. Sci Rep 2017, 7, 8454, doi: 10.1038/s41598-017-09085-5.

32. Metzdorf I.P., Lima Junior M.S. da C., Matos M.C., de Souza Filho A.F., Tsujisaki R.A. de S., Franco K.G., Shapiro J.T., Borges F. de A.: Molecular characterization of Leishmania infantum in domestic cats in a region of Brazil endemic for human and canine visceral leishmaniasis. Acta Tropica 2017, 166, 121-125, 10.1016/j.actatropica.2016.11.013.

33. Moreno J., Alvar J.: Canine leishmaniasis: epidemiological risk and the experimental model. Trends Parasitol 2002, 18, 399-405, doi: 10.1016/s1471-4922(02)02347-4

34. Neto S.Y., Silva F.G.S. da, Souto D.E.P., Faria A.R., Andrade H.M.de, Luz R. de C.S., Kubota L.T., Damos F.S.: Photoelectrochemical immunodiagnosis of canine leishmaniasis using cadmium-sulfide-sensitized zinc oxide modified with 
synthetic peptides. Electrochem Communic 2017, 82, 75-79, doi: 10.1016/j.elecom.2017.07.027.

35. Otranto D., Dantas Torres F.: The prevention of canine leishmaniasis and its impact on public health. Trends Parasitol 2013, 29, 339-345, doi:10.1016/j.pt.2013.05.003.

36. Ramírez J.D., Hernández C., León C.M., Ayala M.S., Flórez C., González C.: Taxonomy, diversity, temporal and geographical distribution of cutaneous leishmaniasis in Colombia: a retrospective study. Sci Rep 2016, 6, 28266, doi: 10.1038/srep28266.

37. Reguera R.M., Morán M., Pérez-Pertejo Y., García Estrada C., Balaña-Fouce R.: Current status on prevention and treatment of canine leishmaniasis. Vet Parasitol 2016, 227, 98-114, doi:10.1016/j.vetpar.2016.07.011.

38. Scott P., Novais F.O.: Cutaneous leishmaniasis: immune responses in protection and pathogenesis. Nat Rev Immunol 2016, 16, 581-592. doi: 10.1038/nri.2016.72.

39. Selder R., Weber K., Bergmann M., Geisweid K., Hartmann K.: Sensitivity and specificity of an in-clinic point-of-care PCR test for the diagnosis of canine leishmaniasis. Vet $\mathrm{J} 2018 ; 232$, 46-51, doi:10.1016/j.tvj1.2017.12.006.

40. Semião-Santos S.J., el Harith A., Ferreira E., Pires C.A., Sousa C., Gusmão R.: Evora district as a new focus for canine leishmaniasis in Portugal. Parasitol Res 1995, 81, 235-239.
41. Shokri A., Fakhar M., Teshnizi S.H.: Canine visceral leishmaniasis in Iran: a systematic review and meta-analysis. Acta Trop 2016, 65, 76-89, doi: 10.1016/j.actatropica.2016.08.020.

42. Tafuri W.L., Oliveira M.R. de, Melo M.N., Tafuri W.L.: Canine visceral leishmaniosis: a remarkable histopathological picture of one case reported from Brazil. Vet Parasitol 2001, 96, 203-212, doi: 10.1016/s0304-4017(00)00436-2.

43. Valdivia H.O., Almeida L.V., Roatt B.M., Reis-Cunha J.L., Pereira A.A.S., Gontijo C., Fujiwara R.T., Reis A.B., Sanders M.J., Cotton J.A., Bartholomeu D.C.: Comparative genomics of canine-isolated Leishmania (Leishmania) amazonensis from an endemic focus of visceral leishmaniasis in Governador Valadares, southeastern Brazil. Sci Rep 2017, 7, 40804, doi: 10.1038/srep40804.

44. Vélez I.D., Carrillo L.M., López L., Rodríguez E., Robledo S.M.: An epidemic outbreak of canine cutaneous leishmaniasis in Colombia caused by Leishmania braziliensis and Leishmania panamensis. Am J Trop Med Hyg 2012, 86 , 807-811, doi: 10.4269/ajtmh.2012.11-0408.

45. Zambrano-Hernandez P., Ayala-Sotelo M.S., Fuya-Oviedo P., Montenegro-Puentes C.A., Aya-Vanegas N.M., AguileraJaramillo G., Blázquez O., Becerra S., Lozano C., RojasGarcía M.C., Rodríguez-Toro G.: Brote urbano de leishmaniasis visceral en Neiva, Colombia (Urban outbreak of visceral leishmaniasis in Neiva, Colombia - in Spanish). Rev Salud publica $2015,7,514-527$. 